\title{
On the accuracy of simulations of turbulence
}

\author{
M. Wan, ${ }^{1}$ S. Oughton, ${ }^{2}$ S. Servidio, ${ }^{3}$ and W. H. Matthaeus ${ }^{1}$ \\ ${ }^{1}$ Bartol Research Institute, University of Delaware, Newark, Delaware 19716, USA \\ ${ }^{2}$ Department of Mathematics, University of Waikato, Hamilton 3420, New Zealand \\ ${ }^{3}$ Dipartimento di Fisica, Universita' della Calabria, I-87036 Cosenza, Italy
}

(Received 8 June 2010; accepted 12 July 2010; published online 24 August 2010)

\begin{abstract}
The widely recognized issue of adequate spatial resolution in numerical simulations of turbulence is studied in the context of two-dimensional magnetohydrodynamics. The familiar criterion that the dissipation scale should be resolved enables accurate computation of the spectrum, but fails for precise determination of higher-order statistical quantities. Examination of two straightforward diagnostics, the maximum of the kurtosis and the scale-dependent kurtosis, enables the development of simple tests for assessing adequacy of spatial resolution. The efficacy of the tests is confirmed by examining a sample problem, the distribution of magnetic reconnection rates in turbulence. Oversampling the Kolmogorov dissipation scale by a factor of 3 allows accurate computation of the kurtosis, the scale-dependent kurtosis, and the reconnection rates. These tests may provide useful guidance for resolution requirements in many plasma computations involving turbulence and reconnection. (C) 2010 American Institute of Physics. [doi:10.1063/1.3474957]
\end{abstract}

\section{INTRODUCTION}

In simulations of turbulence, there are two familiar figures of merit that are used as a diagnostic of the extent to which a simulation is well-resolved. These are as follows: first, an examination of the behavior of the energy spectrum at wavenumbers near the maximum resolved wavenumber, and second, an estimate of whether the Kolmogorov dissipation wavenumber $k_{\text {diss }}=1 / \eta$ is resolved in the simulation. Sometimes, the condition that the dissipation wavenumber is nearly resolved is viewed as adequate. ${ }^{1-3}$ Recent works have discussed the need for stricter conditions on resolution, ${ }^{4}$ and have examined specifically the resolution required to accurately compute high-order statistical quantities that are of central interest in turbulence theory. ${ }^{5}$ Herein we show that there are alternative, easily calculable, quantities that provide a more discerning test of the simulations well-resolvedness. We also examine the performance of these simple tests in the context of a magnetohydrodynamics (MHD) problem that is very demanding in terms of spatial resolution, namely, the determination of rates of magnetic reconnection in turbulence. ${ }^{6,7}$

In much of the literature involving simulations of hydrodynamic turbulence, a numerical aim is to attain the highest possible Reynolds numbers so that the properties of fully developed or strong turbulence can be studied. This is often motivated by efforts to understand natural systems that have very high Reynolds number flows, or else to examine theoretical issues that pertain to the asymptotic limit of high Reynolds number, where certain universal features may obtain. In pursuit of these goals, simulators typically perform runs which "just" resolve the smallest relevant dynamical scales, arguing that this makes efficient use of available computing resources. For example, this sometimes means that the phenomena at the scale of about $2 \eta$, where $\eta$ is the Kolmogorov dissipation scale, are marginally resolved. As we discuss below, this kind of commonly used resolution is adequate for computing second-order quantities such as the energy spectrum, but underestimates high-order moments of velocity gradients, such as the energy dissipation rate and kurtosis. ${ }^{8}$

The present perspective is directly related to a recent study ${ }^{9}$ in which we found that (very) early time energy transfer in turbulence propagates non-Gaussian statistical features into the small scales. For any resolution, some excitation is propagated to the numerical "wall" represented by the maximum wavenumber. If the resolution is inadequate, the excitation level (Fourier amplitude) near the wall is unrealistically large even though the spectrum may "look" small there. The conservative nonlinear couplings involving these nearwall scales then cause phase errors and a re-Gaussianization (or "thermalization") of the solutions (see, e.g., Refs. 10 and 11). This leads to a perspective in which phase errors may be viewed as more sensitive indicators of resolution than the spectrum itself would be. Furthermore the development and maintenance of non-Gaussian statistics at high wavenumbers appears to be a feature associated with adequacy of spatial resolution. The fact that inadequate resolution is expected to promote thermalization and therefore a reduction of nonGaussianity, motivates the present study, which is geared toward establishing a class of simple tests that are useful in determining whether a particular simulation is "wellresolved."

We begin with the hypothesis that stable computation of the fourth-order moments is desirable. A more sensitive test involving scale-dependent kurtosis is also found to be indicative. To understand the utility of these ideas we examine a test problem in which the quantity of interest is the distribution of magnetic reconnection rates in MHD turbulence. Since many reconnection sites occur, we are particularly interested in the conditions on spatial resolution that must be attained to accurately compute the tail of this distribution, because this tail measures the likelihood of the highest rates of reconnection. We find that the same conditions for accu- 
rately computing the fourth-order moments also give rise to accurate computation of reconnection rates.

The two-dimensional (2D) MHD model that we employ in the following analysis is chosen because it remains of importance in space physics and various applications of plasma physics. ${ }^{12,13}$ It also permits many computational runs to be carried out at widely varying spatial resolutions. As is well-known, 2D MHD exhibits a direct cascade of energy and produces more small-scale structure than 2D hydrodynamics. In these ways, it is closer to three-dimensional (3D) MHD turbulence than 2D hydrodynamics is to its $3 \mathrm{D}$ counterpart. $^{14}$

The remainder of the paper is structured as follows. In Sec. II, we give details of the equations solved and the numerical approach. Section III considers code accuracy issues for a set of runs with fixed resolution but varying the Reynolds number. The complementary situation of runs with fixed Reynolds numbers but varying resolution is examined in Sec. IV. Several accuracy criteria are proposed there, and then their usefulness in an example applicationreconnection rates-is considered in Sec. V. Section VI closes the paper.

\section{EQUATIONS AND NUMERICAL METHOD}

The equations of incompressible 2D MHD can be written in terms of the vorticity $\omega=(\nabla \times v) \cdot \hat{z}$ and the vector potential $a$,

$$
\begin{aligned}
& \frac{\partial \omega}{\partial t}=\nabla \cdot[\boldsymbol{b} j-\boldsymbol{v} \omega]+\nu \nabla^{2} \omega, \\
& \frac{\partial a}{\partial t}=-\boldsymbol{v} \cdot \nabla a+\mu \nabla^{2} a .
\end{aligned}
$$

Here, $\boldsymbol{v}$ is the fluid velocity, $\boldsymbol{b}=\nabla a \times \hat{z}$ is the magnetic field, and $j=-\nabla^{2} a$ is the electric current density; these are functions of the Cartesian coordinates $x$ and $y$, and time $t$. The components of $\boldsymbol{v}$ and $\boldsymbol{b}$ lie in the $(x, y)$ plane. The viscous and resistive dissipation coefficients are, respectively, $\nu$ and $\mu$, and are equivalent to reciprocal Reynolds numbers with the normalization employed.

We solve the Fourier-space version of these equations via a Galerkin spectral method, with $N$ Fourier modes in each Cartesian direction. ${ }^{15}$ Time advancement occurs via a second-order Runge-Kutta algorithm. All runs are performed with $\nu=\mu$ and are unforced.

Several important parameters are relevant to our study. The runs are dealiased, using either 2/3-rule dealiasing with the maximum retained dynamical wavenumber $k_{\max }=N / 3$ (runs in Table I), or the Orszag-Patterson approach ${ }^{16}$ with $k_{\max }=\sqrt{2} N / 3$ and phase-shift dealiasing (runs in Table II). Another dealiasing scheme (results not shown) provides a smooth truncation intermediate to the two cases shown here. $^{17}$

Since $\nu=\mu$ herein, the dissipation wavenumber (reciprocal of the Kolmogorov scale $\eta$ ) is defined as
TABLE I. Parameters for simulation set 1 (fixed resolution, varying $\nu$ ). Here $k_{\text {diss }}$ means the maximum value of $k_{\text {diss }}(t)$. The initially excited Fourier modes have wavenumbers $k \in[3,8]$ and use $k_{0}=5$ in the spectral shape function. The time step is $1 / 5000$. Note that runs $4-7$ are definitely underresolved.

\begin{tabular}{lccccc}
\hline \hline Run & Grid & Re & $k_{\max }$ & $k_{\text {diss }}$ & $\frac{k_{\max }}{k_{\text {diss }}}$ \\
\hline 1 & $1024^{2}$ & 600 & 341 & 98 & 3.5 \\
2 & $1024^{2}$ & 800 & 341 & 118 & 2.9 \\
3 & $1024^{2}$ & 1000 & 341 & 136 & 2.5 \\
4 & $1024^{2}$ & 1500 & 341 & 179 & 1.9 \\
5 & $1024^{2}$ & 2000 & 341 & 217 & 1.6 \\
6 & $1024^{2}$ & 3000 & 341 & 287 & 1.2 \\
7 & $1024^{2}$ & 3600 & 341 & 325 & 1.05 \\
8 & $1024^{2}$ & $\infty$ & 341 & $\cdots$ & $\cdots$ \\
\hline \hline
\end{tabular}

$$
k_{\mathrm{diss}}(t)=\left(\frac{\epsilon}{\nu^{3}}\right)^{1 / 4} \equiv \frac{\left\langle\omega^{2}+j^{2}\right\rangle^{1 / 4}}{\sqrt{\nu}},
$$

where $\langle\cdots\rangle$ denotes spatial averaging and $\epsilon$ is the average rate of energy dissipation. The ratio $r(t) \equiv k_{\max } / k_{\text {diss }}(t)$ $\equiv k_{\max } \eta$ can be interpreted as a measure of adequate resolution of the dissipation scale with larger values indicating better resolution. Below, we make regular use of $r(t)$ as an organizing parameter for the simulations, evaluating it at several distinct times. A closely related measure of resolution is the ratio $\Delta x / \eta,{ }^{5}$ where $\Delta x \equiv 2 \pi / N$ is the grid scale.

The initial spectra for the runs are taken to be proportional to $1 /\left[1+\left(k / k_{0}\right)^{8 / 3}\right]$, where only Fourier modes within a restricted band of wavenumbers $k=|\boldsymbol{k}|$ are excited. Gaussian random numbers (mean of zero, variance unity) are used to assign the phases of the Fourier modes. The particular bands and values of $k_{0}$ employed are given in the captions of the two tables below. The total kinetic energy and the total magnetic energy are both equal to 0.5 at $t=0$. In all runs, the normalized cross helicity, $\sigma_{c}=2\langle\boldsymbol{v} \cdot \boldsymbol{b}\rangle /\left\langle\boldsymbol{v}^{2}+\boldsymbol{b}^{2}\right\rangle$, is initially small and remains so throughout the runs. We would like to emphasize that the codes are well-tested and conserve extremely well all the "rugged invariants," namely, total energy, cross helicity, and $\left\langle a^{2}\right\rangle$. The ideal conservation of qua-

TABLE II. Parameters for simulation set 2, which all have $\nu=\mu=1 / 2000$. The initially excited Fourier modes have $5 \leq|\boldsymbol{k}| \leq 30$ with $k_{0}=10$. Again, $k_{\text {diss }}$ means the maximum value of $k_{\text {diss }}(t)$. Runs 1-3 are definitely underresolved.

\begin{tabular}{lccccc}
\hline \hline Run & Grid & Re & $k_{\max }$ & $k_{\text {diss }}$ & $\frac{k_{\max }}{k_{\text {diss }}}$ \\
\hline 1 & $128^{2}$ & 2000 & 60 & 221 & 0.27 \\
2 & $256^{2}$ & 2000 & 120 & 242 & 0.50 \\
3 & $512^{2}$ & 2000 & 241 & 250 & 0.96 \\
4 & $1024^{2}$ & 2000 & 482 & 251 & 1.9 \\
5 & $1536^{2}$ & 2000 & 724 & 251 & 2.9 \\
6 & $2048^{2}$ & 2000 & 965 & 251 & 3.9 \\
7 & $4096^{2}$ & 2000 & 1930 & 251 & 7.7 \\
8 & $8192^{2}$ & 2000 & 3861 & 251 & 15.4 \\
\hline \hline
\end{tabular}



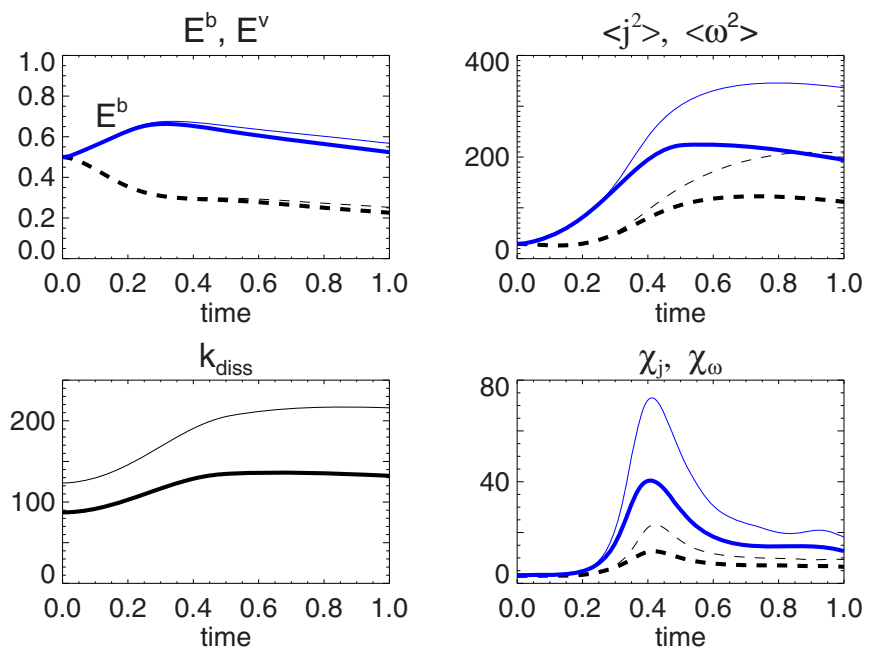

FIG. 1. (Color online) Time histories for energies, $k_{\text {diss }}$, and variances and kurtoses of $j$ and $\omega$ for two $N=1024$ runs. Thick curves: $\nu=1 / 1000$ (ostensibly adequately resolved). Thin curves: $\nu=1 / 2000$ (under-resolved). Except in the $k_{\text {diss }}$ panel, quantities based on the magnetic (velocity) field are shown using solid (dashed) lines.

dratic quantities is another important requirement for simulations of turbulence, and a high level of accuracy can be attained with the dealiased spectral method simulations employed here. For example, for the ideal $1024^{2}$ run in Table $\mathrm{I}$, the rugged invariants are conserved to better than $0.02 \%$ at $t \sim 1$.

\section{FIXED RESOLUTION, VARYING REYNOLDS NUMBER}

To illustrate the basic physics of the system for changing adequacy of resolution, we examine a set of simulations with the code resolution fixed at $N_{x}=N_{y}=N=1024$. The Reynolds numbers of the runs are varied by employing the following values of $1 / \nu: 600,800,1000,1500,2000,3000$, and 3600 . We also carry out a nondissipative run, which formally has $1 / \nu=\infty$, although, of course, this is not equivalent to the $\mathrm{Re} \rightarrow \infty$ limit. The main run parameters are listed in Table I.

\section{A. Globals and kurtosis}

Figure 1 shows the evolution of several global quantities, including the kurtosis of $j$ and $\omega$, for two of the $N$ $=1024$ runs. Using the familiar standard criterion that $k_{\text {diss }}$ $<k_{\max }$, one would judge the $\nu=1 / 1000$ run (thick lines) to be well-resolved, and similarly for the $\nu=1 / 2000$ run, although less so. The behavior of the globals shows the expected tendencies as one moves toward higher Reynolds number. For example, the mean-square vorticity (enstrophy) and mean-square current density attain higher maximum values in the higher Reynolds number case, because the smallest dynamically relevant length scale is smaller, as indicated by the decreased $\eta=1 / k_{\text {diss }}$ (Table I).

The behavior of the energies in the two runs is similar enough that it would be difficult to decide which, if any, of the simulations was under-resolved. The situation is essentially the same for the other quantities shown, with the two runs showing qualitatively similar behavior, although with

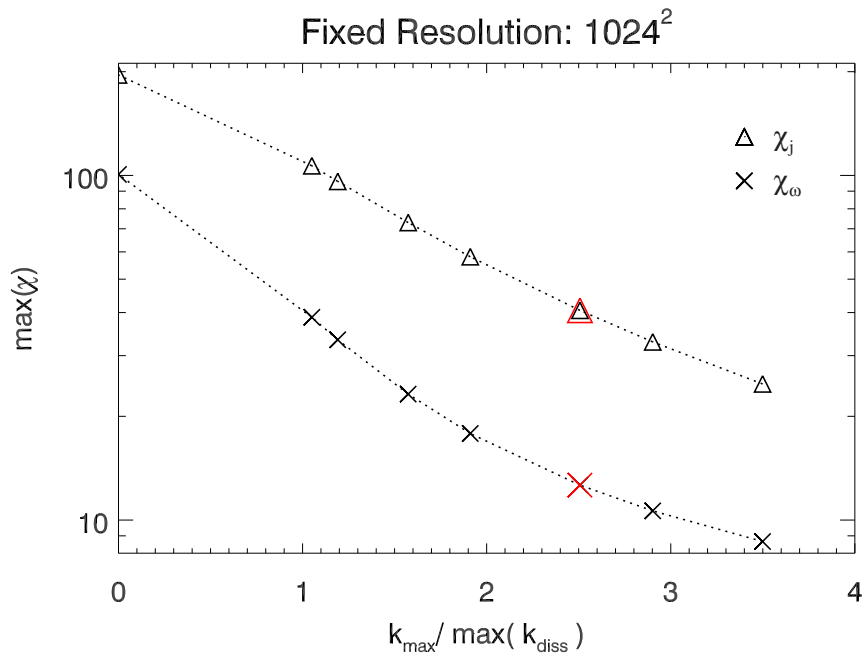

FIG. 2. (Color online) Maximum kurtosis of $j$ (triangles) and $\omega$ (crosses) as a function of $k_{\max } / k_{\text {diss }}$, for the fixed resolution run set. From right to left, the symbols correspond to the run with $1 / \nu=600,800,1000,1500,2000,3000$, 3600 , and $\infty \equiv$ ideal). Here, $k_{\text {diss }}$ is evaluated at the time of maximum $\chi_{j}$. The two larger symbols identify the $1 / \nu=1000$ run, which is close to being well-resolved.

significant quantitative differences. The kurtosis $\chi_{j}$ $=\left\langle j^{4}\right\rangle /\left\langle j^{2}\right\rangle^{2}$ of $j$ is one measure of a field's degree of departure from Gaussianity; Gaussian distributed quantities necessarily have $\chi=3$. As is well-known for decaying 2D MHD turbulence, $\chi_{j}$ first increases to a relatively sharply peaked maximum, and then decreases before plateauing at a roughly constant (non-Gaussian) value, as seen in Fig. 1. The higher values of kurtosis for higher Reynolds number runs indicate the presence of sharper concentrations in $x$-space, that is, coherent structures of finer scale. Below, we will use the maximum of $\chi_{j}$ and the time it occurs as diagnostics.

Figure 2 shows how the maximum value of $\chi$ (for both $j$ and $\omega$ ) varies as a function of $r=k_{\max } / k_{\text {diss }}$ as $\nu$ varies. The maximum kurtosis increases smoothly as $\nu$ (and thus $r$ ) decreases, reaching its maximum in the ideal $(\nu=0)$ run. Unfortunately, it seems that the diagnostic value of this behavior is limited. A particular issue is that the time variation of $\chi$ in ideal runs and well-resolved dissipative runs can be similar, but for quite different reasons (see Ref. 9). Therefore simply examining the behavior of maximum kurtosis at fixed resolution is not a sensitive diagnostic.

\section{B. Spectra}

Another approach to evaluating the quality of the spatial resolution is to view the wavenumber spectra for the total (kinetic plus magnetic) energy. Figure 3 shows energy spectra from the simulations listed in Table I. In all cases, the spectra are from the time closest to that of the run's maximum kurtosis, whether it is later or earlier. ${ }^{18}$

As Fig. 3 indicates, these simulations yield reasonable looking spectra. Indeed, they all overlay each other at low enough $k$. It is also quite possible to obtain what looks like a clean power-law inertial range. The differences at high $k$ are 


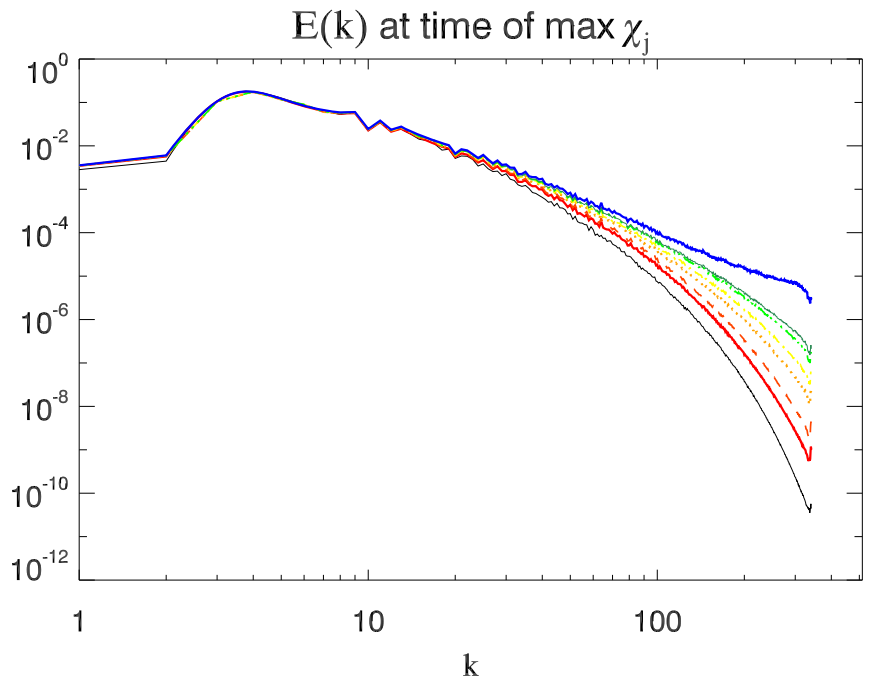

FIG. 3. (Color online) Total energy spectra at or near the time of maximum kurtosis for the runs in Table I (fixed resolution, varying $\nu$ ). The curves are in order of run number with run 1 the lowest curve.

expected, and there is no significant "build-up" of energy near the resolution limit, at this early time in the runs, except perhaps for the ideal run.

\section{Scale-dependent kurtosis}

In some ways, an even more revealing quantity is the scale-dependent (or filtered) kurtosis, $\chi_{j}^{>}\left(k_{c}\right)$, defined as the kurtosis of the high-pass filtered $j(x)$. In other words, the Fourier components of $j$ with wavenumbers $k<k_{c}$ are zeroed, and then the kurtosis of the resulting field is calculated. This quantity measures a characteristic that is very close to what Frisch ${ }^{19}$ discussed as the defining property of spatial intermittency. In addition, we have recently shown ${ }^{9}$ that $\chi^{>}\left(k_{c}\right)$ provides a direct measure of the thermalization of the phases, which occurs due to conservative interactions with the modes near the Fourier space cutoff at $k_{\max }$.

In Fig. 4, we plot the high-pass filtered kurtosis for simulation set 1. It is clear that for each run $\chi^{>}\left(k_{c}\right)$ increases with the cutoff $k_{c}$, indicating some generation of coherent structures, or "intermittency." ${ }^{, 20}$ What is interesting in the present context is that the higher Reynolds number cases do not attain greater maxima at high cutoff wavenumber. [Note that as $k_{c} \rightarrow k_{\max }$, the number of modes in the kurtosis calculation decreases toward a small limiting integer, implying a sharp decrease of $\chi^{>}\left(k_{c}\right)$ to values near 1.] This may lead us to suspect that the runs in Table I with the highest Reynolds numbers may have impending problems with resolution that have not yet had an impact on diagnostics such as the spectrum. In summary, although everything we have examined so far seems to behave reasonably, we have not yet identified any clear indicators of how well-resolved each of these runs is.

\section{A STUDY OF THE QUALITY OF RESOLUTION}

Having seen in Sec. III that simulations at fixed resolution and varying Reynolds number did not provide any obvious means of identifying how well-resolved the runs
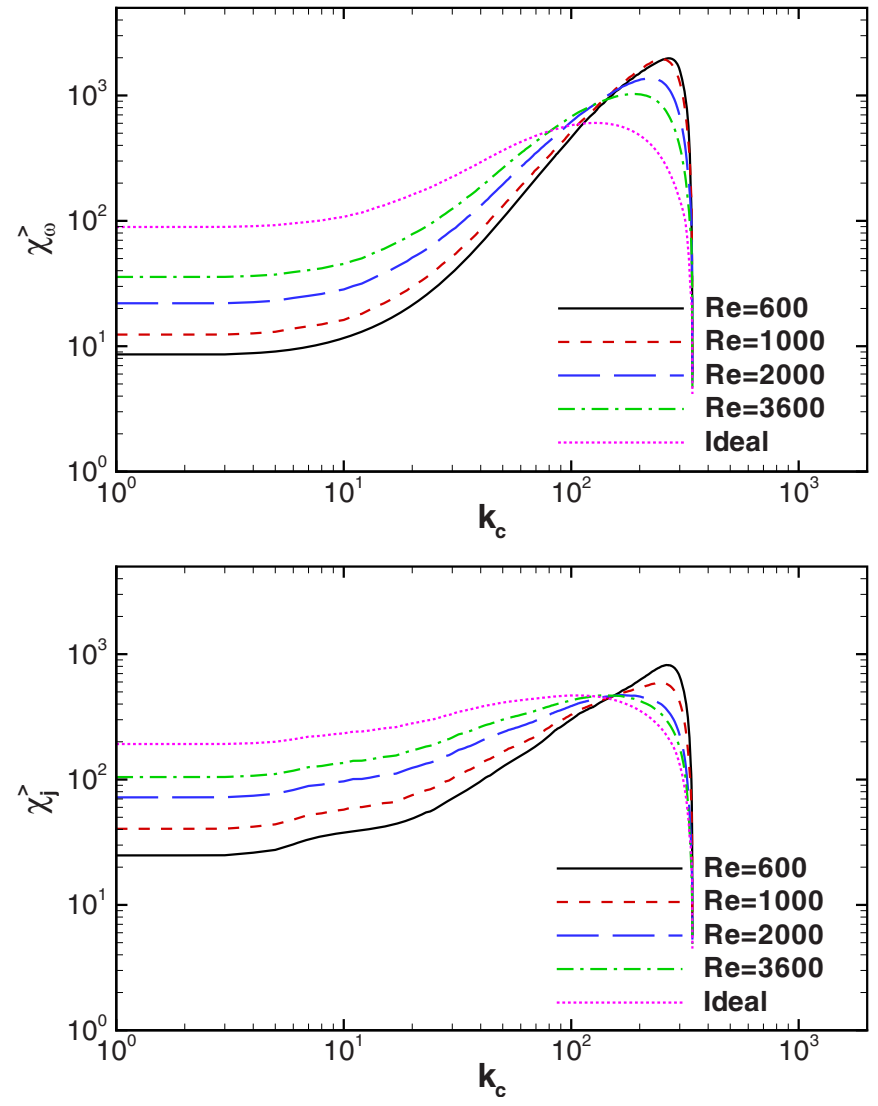

FIG. 4. (Color online) High-pass filtered kurtosis of vorticity (top) and of current density (bottom) for the runs of simulation set 1 (see Table I). $k_{c}$ is the cutoff wavenumber for the high-pass filter.

were, we now investigate the complementary problem. That is, we consider a new series of runs (Table II) in which the initial physical parameters-including the Reynolds numbers-are fixed, and the numerical resolution is varied. Using this set of runs, we will examine conditions for wellresolvedness, and then, in Sec. V, test the ideas by study of an ostensibly unrelated physical property, the rate of magnetic reconnection.

\section{A. Spectra}

Energy spectra for the runs listed in Table II are displayed in Fig. 5. For each run, the spectrum is computed at (or very near) the time of maximum $\chi_{j}$. Panel (a) shows that all the spectra agree well. Indeed, although the spectra cutoff at different wavenumbers (because of the different run resolutions), the curves nearly overlay each other over the full range of overlapping $k$, with only small discrepancies in the lower resolution runs near their maximum retained wavenumber. Panel (b) is a zoom of panel (a) for the lower $k$ values, revealing that to a good approximation all the resolutions used-including cases that will be argued below to be under-resolved-provide an accurate energy spectrum near the time of maximum $\chi_{j}$, at low enough $k$. The importance of this comparison is that an examination of the "quality" of an energy spectrum is a frequently encountered approach to 

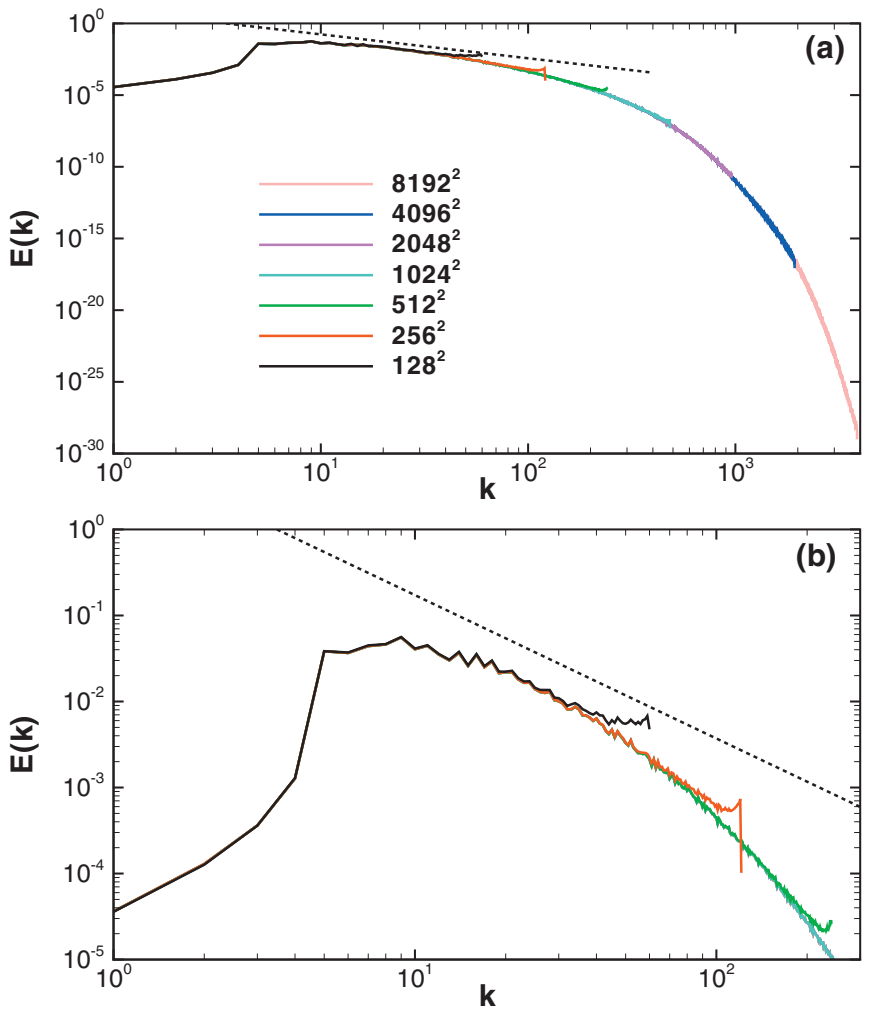

FIG. 5. (Color) (a) Energy spectra at the time of maximum $\chi_{j}$ for all runs in simulation set 2 (fixed $\nu$, varying $N$ ). A $k^{-5 / 3}$ powerlaw (dotted) is shown for comparison. (b) A zoom of panel (a) to highlight the agreement between runs at lower $k$. The $1536^{2}$ run is not plotted (as also in Figs. 8 and 10) to avoid clutter.

heuristically evaluate the quality of spatial resolution. However we now show that spectra can be reasonably accurate even when other quantities are not.

\section{B. Kurtosis}

The runs listed in Table II exhibit an interesting range of behavior in terms of their fourth-order statistics. Below we will show that this behavior is a useful tool for ascertaining the quality of the spatial resolution of the simulations.

We plot in Fig. 6 the time dependence of the kurtosis of the current density, $\chi_{j}$, for the simulations of Table II. For all

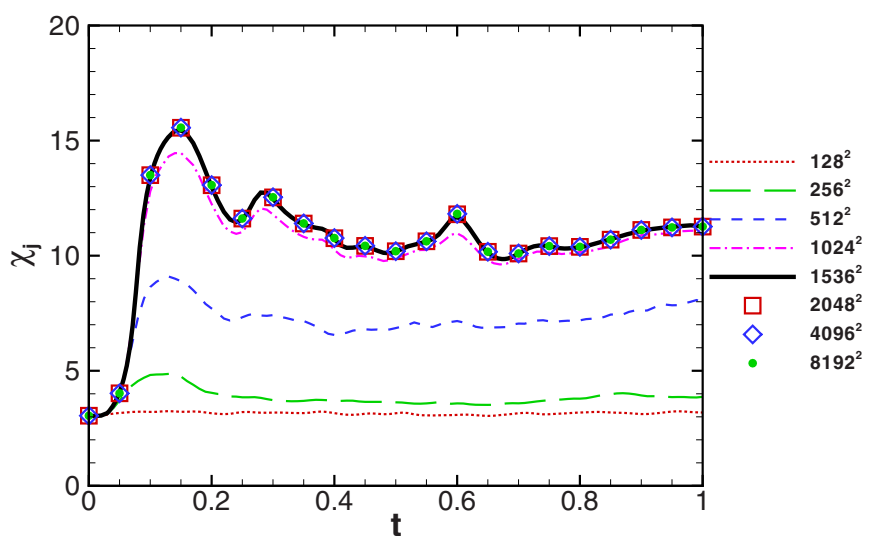

FIG. 6. (Color online) The time evolution of kurtosis of the current density for the simulation set 2 runs.

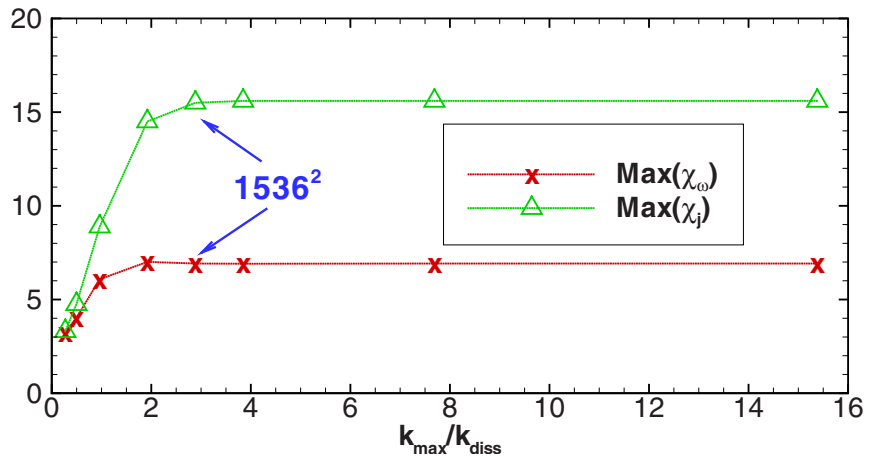

FIG. 7. (Color online) Maximum kurtosis as a function of $k_{\max } / k_{\mathrm{diss}}$ for the simulation set 2 runs.

runs except the $128^{2}$ one, $\chi_{j}$ first increases to maximum, and then decreases before plateauing at a roughly constant (nonGaussian) value, similar to the results in Fig. 1. It would seem that accurate (i.e., resolution independent) behavior occurs once $N \geqq 1536$.

In Fig. 7, we plot the maximum kurtosis as a function of $r=k_{\max } / k_{\text {diss }}$ (evaluated when $k_{\text {diss }}$ takes its maximum). For the lower resolution runs, the maximum kurtosis increases rapidly with $r$, with $\max \left(\chi_{j}\right)$ increasing by about a factor of 3 as the resolution goes from $256^{2}$ to $1024^{2}$. Obviously in this range of resolutions, the solution is not very well-determined in terms of fourth-order statistics, even though the spectra are very nearly identical (Fig. 5). However, a clear saturation occurs once $r$ becomes large enough. For the fixed value of $\nu$ used in these runs, resolution of $N=2048$ is more than adequate. In fact, at $N=1536$ (1024), the kurtosis of $j$ is only $0.65 \%(6.5 \%)$ lower than in the fully resolved cases. Either of these resolutions may represent an acceptable level of accuracy, depending upon the application.

Note that although the effect is qualitatively the same for both $\max \left(\chi_{j}\right)$ and $\max \left(\chi_{\omega}\right)$, it is much more pronounced for the former. Presumably because $j$ is the more singular (that is, more strongly non-Gaussian) quantity in incompressible 2D MHD.

Very similar behavior is seen if the (normalized) sixth moment is used in place of the kurtosis (not shown). This suggests that in many cases of interest, a necessary condition for a well-resolved simulation is that it produces an accurate value for the maximum kurtosis of $j$, since the current density $j$ is often the "most singular" fourth-order quantity for decaying 2D MHD.

\section{Scale-dependent kurtosis}

A moment's consideration reveals that the final statement of Sec. IV B cannot be strictly correct, even though it may be useful in a practical sense. This is due to the fact that, at high Reynolds numbers, the most singular structures in the system will always require the finest resolution. The lack of complete resolution of such a rare structure will cause some very high order moments to be inaccurately computed from a simulation. Here we show, using the simple technique of evaluation of the scale-dependent kurtosis, that the very high wavenumber structures become increasingly coherent (or 

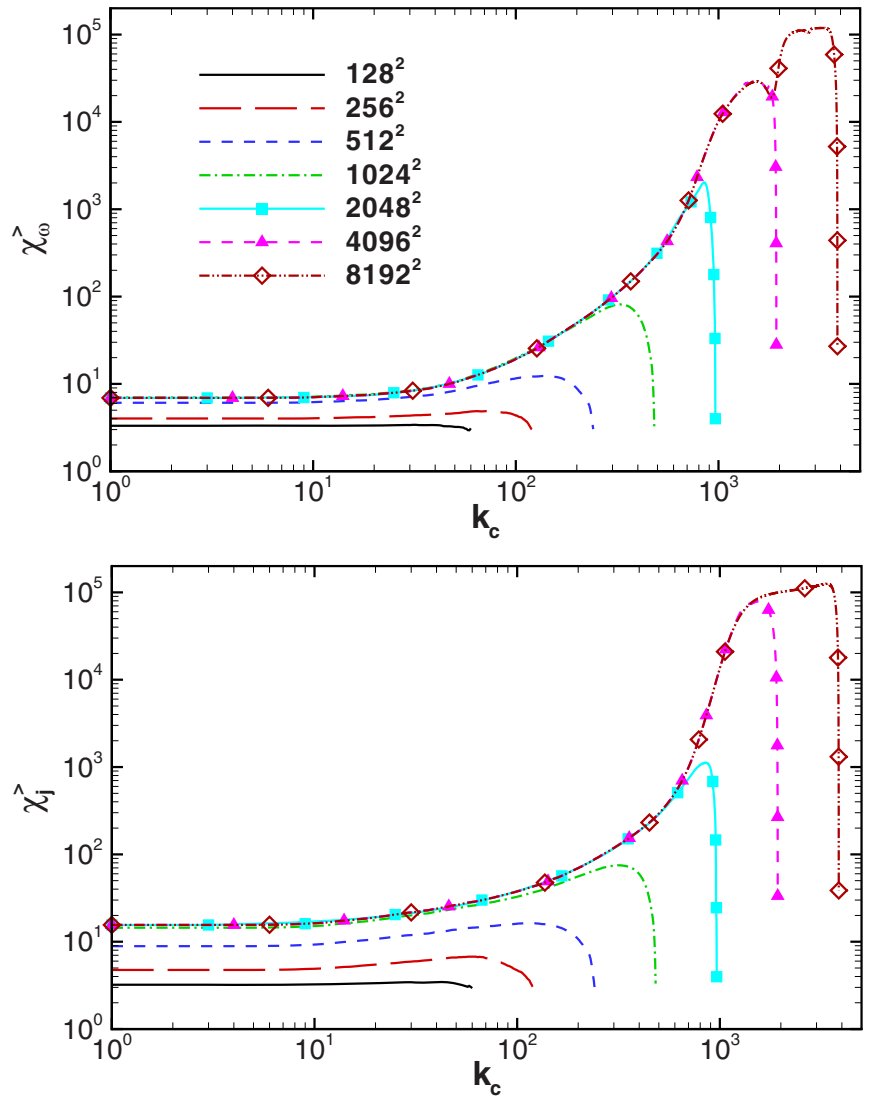

FIG. 8. (Color online) High-pass filtered kurtosis of vorticity (top) and of current density (bottom), for the simulation set listed in Table II, computed at the time of $\max \left(\chi_{j}\right)$. Note that this scale-dependent kurtosis increases monotonically for all wavenumbers that have significant energy, and that are also well-separated from the highest resolved wavenumber $k_{\max }$. The rapid decrease near $k_{\max }$ is associated with the absence of couplings to scales with $k>k_{\max }$, which is itself an artifact of the Fourier space truncation. The anomalous behavior at very high $k$ in the highest resolution case is caused by issues of numerical precision as there is very little energy in that part of the spectrum (see Fig. 5).

"intermittent") even if they possess almost no energy. Therefore adequate resolution of moments of very high order may admit further challenges. ${ }^{4,5}$

In Fig. 8, we plot the high-pass filtered kurtosis of the electric current density and the vorticity for the Table II simulation set (fixed viscosity). The monotonic increase for all dynamically relevant wavenumbers is a signal of intermittency (see Ref. 19). Note that according to this basic definition of intermittency, the $128^{2}$ run-obviously having inadequate resolution-is flat and therefore lacks intermittency almost completely. For the better resolved runs, $\chi^{>}\left(k_{c}\right)$ is large at high $k_{c}$; however, in terms of the full (unfiltered) field, this will only affect its very high-order moments.

Significantly, we see that $\chi^{>}\left(k_{c}\right)$ saturates to a stable value at the lower $k_{c}$ values $(\approx 15.6$ for current and $\approx 6.9$ for vorticity), as resolution is increased. The lowest $k$-filtered kurtosis for the $128^{2}$ runs is almost at the Gaussian value of 3. In contrast, for example, the filtered kurtosis at $k_{c}=100$ becomes very accurate for resolutions greater than $1024^{2}$. For resolution of $2048^{2}$ the filtered kurtosis of both the current density and the vorticity are accurate up to wavenumbers of at least $k_{c}=500$. This implies that the filtered kurtosis at the dissipation wavenumber $\left(k_{\text {diss }} \approx 251\right)$ becomes determined accurately somewhere between the resolutions of $1024^{2}$ and $2048^{2}$. If we compare these values with the accuracy of resolution results deduced from Fig. 7, we see that this is also the range of resolutions that places the computed solutions securely on the plateau of stable values of maximum (unfiltered) kurtosis. (Note that the saturation of values in Fig. 7 is essentially the same as the saturation at $k_{c}=1$ in Fig. 8.)

Based on this line of reasoning, we propose that an effective test of adequate resolution of a turbulence simulation is to ascertain that the maximum kurtosis has attained its stable plateau value, as described above in connection with Fig. 7. This should ensure that most moments of interest are well-determined, and that the kurtosis of fluctuations at the dissipation scale (and somewhat beyond) is known accurately. It is worthwhile pointing out that calculation of $\chi^{>}\left(k_{c}\right)$ is considerably more expensive than that of $\max (\chi)$.

\section{A TEST CASE: RECONNECTION RATES}

As we have seen above, the adequacy of spatial resolution directly influences computation of quantities related to intermittency. In such cases, one would typically expect simulationists to take care to meet the requirements outlined above. However the subtleties of assessing sufficient numerical resolution are also relevant to computation of other physical quantities of importance. As a test case, we consider here the familiar and physically important phenomenon of magnetic reconnection (see, e.g., Refs. 13 and 21).

In reconnection, regions of oppositely directed magnetic field interact with one another in a thin boundary layer, giving rise to changes in magnetic topology, and conversion of magnetic energy into flows and heat. ${ }^{22,23}$ In plasma physics the small-scale phenomena near a reconnection layer (or "site") are expected to depart from a simple MHD description. However, even in MHD the reconnection layers are small (thin) and represent a kind of coherent structure supported by nonlinear couplings across a wide range of length scales. A particularly delicate issue is the computation of the rate of reconnection, identified with the electric field at the $\mathrm{X}$-type neutral point within the reconnection layer. Here we will adopt the study of reconnection rates in turbulence as a test case for application of the measures of spatial resolution considered in Sec. IV.

A recent investigation computed reconnection rates at large numbers of X-points in incompressible 2D MHD turbulence. ${ }^{6,7}$ We employ techniques similar to those developed in these earlier studies, to evaluate quantitatively the impact of varying spatial resolution on the probability distribution of reconnection rates. As is standard, we use the terminology magnetic island to mean a region where the contours of the magnetic potential $a(x, y)$ are closed.

To find the magnetic reconnection sites in the turbulent system, we need to examine the topography of the magnetic potential $a(x, y)$. In particular we need to identify the neutral points - points where $\nabla a=0$ - and their nature. A useful tool in this regard is the Hessian matrix of $a$, defined as 


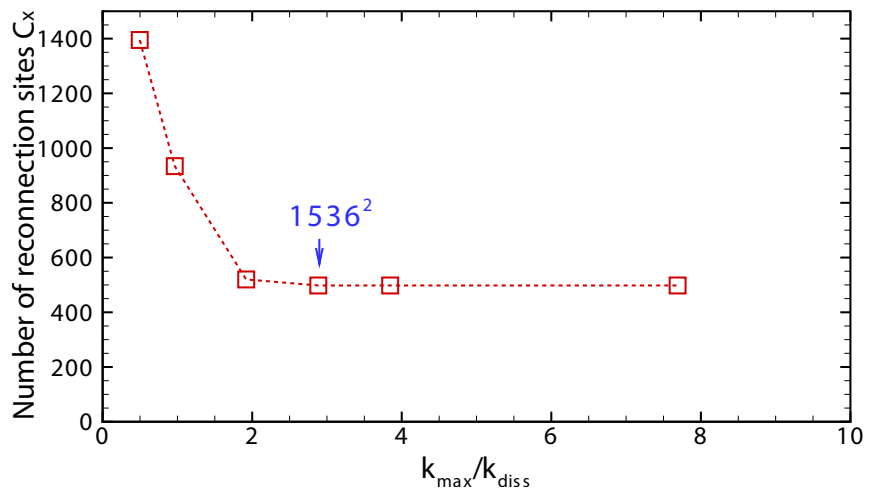

FIG. 9. (Color online) Number of reconnection sites $C_{X}$ as a function of $k_{\max } / k_{\text {diss }}$ for runs listed in Table II (left to right: $256^{2}, 512^{2}, 1024^{2}, 1536^{2}$, $2048^{2}$, and $4096^{2}$ ). For each run, the number of sites is calculated at (or very near) the time of $\max \left(\chi_{j}\right)$.

$$
H_{i j}^{a}(x)=\frac{\partial^{2} a}{\partial x_{i} \partial x_{j}} .
$$

At each neutral point, we calculate the eigenvalues of $H_{i j}^{a}(x)$. If both eigenvalues are positive (negative), the point is a local minimum (maximum) of $a$, with both cases generically referred to as O-points. If the eigenvalues are of mixed signs, it is a saddle point, also termed an X-point. As a technical point, note that for each run the $a(x, y)$ is first interpolated onto a standard grid with a resolution of $N=8192$, and it is this $a$ that is used for determining the positions and natures of the critical points. This enhances the validity of comparisons between the different resolution runs.

Having obtained details on all the critical points in the domain, the reconnection rate of two islands can be determined by computing the electric field at the X-point between the islands. This is related to the fact that the magnetic flux through a closed 2D island is equal to the integral of the component of magnetic field normal to any contour connecting the central O-point with any other specified point. Choosing that point to be an X-point bounding the island, we find that the flux in the island is just $a$ (O-point) $-a$ (X-point). Magnetic flux is always lost at the $\mathrm{O}$-point in a dissipative system so the time rate of change of the magnetic flux due to activity at the X-point is $-\partial a(\mathrm{X}$-point $) / \partial t$ $=E_{z}$ (X-point). The latter step follows from the Ohm's law expression for the electric field

$$
\boldsymbol{E}=-\boldsymbol{v} \times \boldsymbol{b}+R_{\mu}^{-1} \boldsymbol{j},
$$

which in 2D gives only an out-of-plane component, $E_{z}$. At an $\mathrm{X}$-type neutral point $\boldsymbol{b}$ is zero so that $E_{z}(\mathrm{X}$-point $)=R_{\mu}^{-1} j_{z}$. Here, $R_{\mu} \propto 1 / \mu$ is the magnetic Reynolds number. Below we denote the reconnection rate at the $i$ th $\mathrm{X}$-point as $E_{X, i}$ $\equiv E_{z}(\mathrm{X}$-point $)$.

To carry out these tests we use the sequence of runs listed in Table II and examine the resolution dependence of several reconnection-related quantities. First, using the above methods, we compute the total number of X-points found in each run at (or very near) the time of maximum $\chi_{j}$, denoting the total as $C_{X}$. Figure 9 displays these counts as a function of $k_{\max } / k_{\text {diss }}$. We see, as was reported elsewhere, ${ }^{7}$ that under-
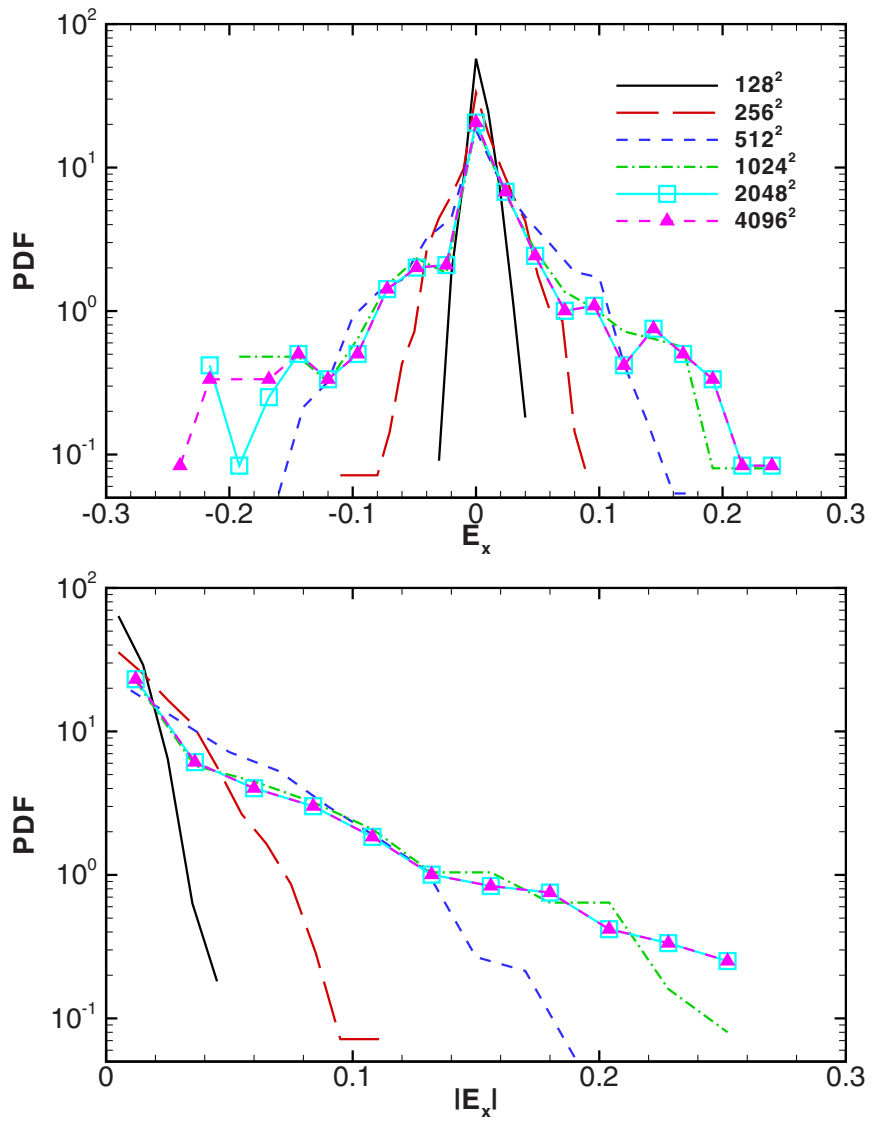

FIG. 10. (Color online) PDFs of the reconnection rate $E_{X}$ (top) and $\left|E_{X}\right|$ (bottom) for the runs in simulation set 2 , near the time of $\max \left(\chi_{j}\right)$.

resolved runs include many additional X-points that are not found in the well-resolved cases. Evidently the phase errors caused by under-resolution imply a lack of intermittency (as discussed in Secs. I and IV), and also a spurious increase in the number of X-points present, due to Gaussianization of the fluctuations (for a fixed spectrum), as was also reported earlier. ${ }^{7}$ However, a clear saturation in $C_{X}$ occurs once $k_{\text {max }} / k_{\text {diss }}$ becomes large enough. For example, although the $1024^{2}$ run is under-resolved according to the criteria considered in Sec. IV, it nonetheless has almost the same number of $\mathrm{X}$-points as the runs with higher resolutions.

Another important feature is the distribution of reconnection rates, i.e., the probability distribution function (PDF) of the electric field at the X-points This distribution of $E_{X}$ values is quite broad and peaked around $E_{X}=0$. In Fig. 10, the PDFs of $E_{X}$ and $\left|E_{X}\right|$ are shown. Comparing the results from different runs, one observes that the PDFs for the under-resolved $128^{2}$ and $256^{2}$ runs have much shorter tails. As the resolution increases, the tails become broader until saturation occurs at about $2048^{2}$. To quantify these differences in the distribution tails, we have calculated various $p$-norms of $E_{X}$, defined ${ }^{24}$ as $\left[\left(1 / C_{X}\right) \sum_{i=1}^{C_{X}} E_{X, i}^{p}\right]^{1 / p}$, and plotted these as a function of $k_{\max } / k_{\text {diss }}$ (Fig. 11). We see that these $p$-norms all saturate with increasing resolution, with those with larger $p$ saturating a little slower, as one would expect. After achieving a resolution of $1536^{2}$ or greater, the 2-norm is very well-determined, and it appears that the $p=4,6$, and 


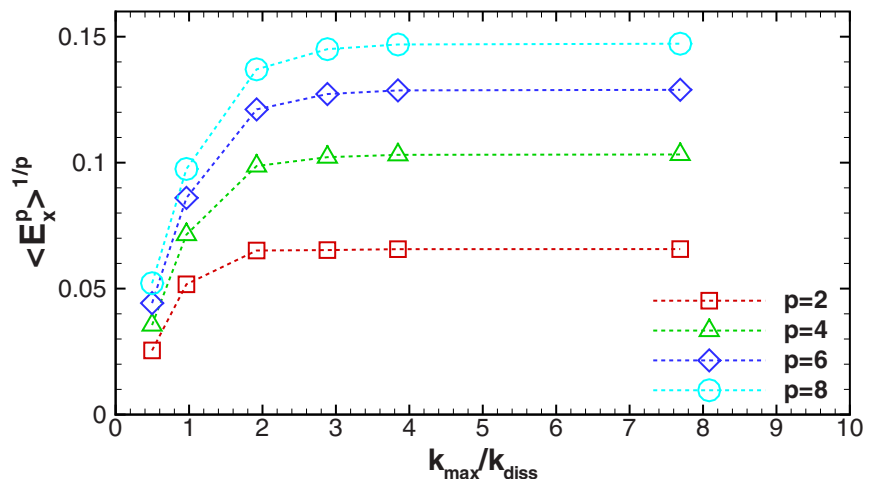

FIG. 11. (Color online) Several $p$-norms of $E_{X}$ for the runs in simulation set 2 , as a function of $k_{\max } / k_{\text {diss }}$. Norms are calculated near the time of $\max \left(\chi_{j}\right)$.

$8 p$-norms of $E_{X}$ are accurate to better than $1 \%$. This degree of accuracy corresponds to $k_{\max } / k_{\mathrm{diss}} \geqslant 3$.

As is evident from Figs. 9 and 10, under-resolving the Kolmogorov scale implies the appearance of many additional reconnection sites, with lower reconnection rates. These spurious $\mathrm{X}$-points generally correspond to fictitious secondary islands that arise from the Gaussian noise/phase errors induced by the under-resolution.

\section{DISCUSSION}

We have reexamined the issue of spatial resolution in simulations of turbulence in the specific context of incompressible 2D MHD spectral method computations. By comparing simulations carried out at varying resolution and at varying Reynolds numbers, we were able to draw several firm conclusions.

(1) The energy spectrum may be well-behaved and reproducible at the larger scales even when $k_{\max } \eta<1$.

(2) Attaining spectral resolution such that $k_{\max } \eta=1$ is not sufficient to ensure the accuracy of higher-order quantities and measures of intermittency.

(3) The maximum of the kurtosis of both the electric current density and the vorticity is underestimated when resolution is inadequate. However, for runs which satisfy $k_{\max } \eta>3$, both $\max \left(\chi_{j}\right)$ and $\max \left(\chi_{\omega}\right)$ are very stable and accurately determined.

(4) The scale-dependent kurtosis of current and vorticity are sharply increasing functions of the high-pass cutoff wavenumber when a run is well-resolved. When a run is under-resolved, these scale-dependent kurtoses are weakly peaked or flat.

(5) Under-resolved values of scale-dependent kurtosis at large scales are lower, and closer to Gaussian, than they should be. As resolution is increased, successively higher ranges of scale-dependent kurtosis become saturated at stable values.

(6) The issues raised above in points (1)-(5) are crucial in numerical studies of structure formation and reconnection that involve phase-sensitive physics.

On the basis of these observations, we propose three criteria for assessing adequate spatial resolution in a spectral method in an incompressible 2D MHD turbulence simulation:

- First, that $k_{\max } \eta>3$;

- second, that the maximum of the kurtosis of current density and vorticity have attained stable "plateau" values;

- third, that the scale-dependent kurtosis at the dissipation scale $\eta$ has attained its stable value.

These conditions are easily translated into practical tests of adequate resolution. The first condition can be evaluated from a single run, using standard estimates of the dissipation scale. ${ }^{25}$ Note that it means that there is half a decade (or more) of wavenumbers above $k_{\text {diss }}$. The condition has been proposed and used before in the literature [e.g., Refs. 5 and 26], but its connection to the accuracy of the kurtosis, for example, has apparently not been explicitly noted. The latter two conditions can be used as a test by comparing two runs with identical parameters and initial data, except the second is done with either higher or lower resolution. An example would be halving the resolution, although it would usually be preferable to double it, if this was feasible.

We also have shown that the above ideas work well in the context of a real research problem, namely, the accurate determination of the distribution of rates of reconnection in 2D MHD turbulence. Here we find that when the above accuracy conditions are satisfied, the distribution of reconnection rates is also well-determined. The reconnection rates are a much more time-consuming calculation so the implementation of the above tests provides a more efficient way to assess the likely accuracy of the results.

It is worthwhile also remarking on the recently improved understanding of the nature of the errors that enter in marginally resolved cases or under-resolved cases, and how they can spoil the quality of results such as reconnection rates. In a recent study, ${ }^{9}$ the focus was on understanding the earlytime ideal couplings that spread excitation rapidly to higher wavenumbers in an initially band-limited cascade of MHD turbulence. We found that the initial spectral transfer to higher $k$ is highly non-Gaussian, and obviously favors dynamical production of the scale-dependent kurtosis that is expected in intermittent turbulence. However, for ideal simulations, this rapid transfer of excitation to high $k$ soon encounters the wall at $k_{\max }$, and for a spectral code the excitation conservatively "reflects" off the wall. Interestingly, errors in the higher-order statistics, which can be thought of as "phase errors," propagate much more rapidly to the wall and back into the solution than do errors in the energy spectrum. This rapidly propagating phase information spoils the higher-order statistics in some cases, while leaving the energy spectrum seemingly well-resolved.

The above description appears to be applicable to the present study as well. Rapidly propagating phase information is more sensitive than the spectrum to the adequacy of spatial resolution. If $k_{\max } \eta$ is not large enough, the higher-order statistics acquire significant errors. Unfortunately, examination of the spectrum is not sufficient to detect this. The study of the distribution of reconnection rates shows explicitly that these issues can greatly affect physical results. In particular, reconnection rates are properties of the thinnest current 
sheets, and presumably the largest rates are associated with the strongest and most intermittent current structures. As such, this problem requires more than just resolution of the spectrum, it also requires accuracy of the higher-order statistics that describe the coherent current (and vorticity) structures. In this way, the criteria and tests that we propose may well be useful in computational problems of this type.

Finally we remark that the present study is complementary to the recent study by Donzis et $a l^{5}$ who were concerned mainly with accuracy of higher-order statistics in hydrodynamic turbulence. The present work can be viewed as applying some of those ideas to MHD in two dimensions, and developing practical tests to be used in 2D MHD applications. What remains unclear at present is how the details of either Donzis et al. ${ }^{5}$ or the present study extrapolate to 3D MHD, or other systems such as 2D hydrodynamics, Hall MHD, etc., including compressible cases. However, we expect that the basic picture discussed above will remain a requirement for accurately computing coherent structures in various fluid systems, and, in particular, that runs would likely need to satisfy $k_{\max } \eta>3$. This, and the other main conclusions of this study, do not appear to depend on details of the method, such as the kind of dealiasing that was employed. ${ }^{16,17}$

\section{ACKNOWLEDGMENTS}

This research is supported in part by the NSF Solar Terrestrial Program under Grant No. ATM0539995, by NASA under the Heliophysics Theory Program Grant No. NNX08AI47G, and by the University of Waikato Strategic Research Investment Fund.

${ }^{1}$ T. Gotoh, D. Fukayama, and T. Nakano, Phys. Fluids 14, 1065 (2002).

${ }^{2}$ Y. Kaneda, T. Ishihara, M. Yokokawa, K. Itakura, and A. Uno, Phys. Fluids 15, L21 (2003).
${ }^{3}$ P. K. Yeung, D. A. Donzis, and K. R. Sreenivasan, Phys. Fluids 17, 081703 (2005).

${ }^{4}$ V. Yakhot and K. R. Sreenivasan, J. Stat. Phys. 121, 823 (2005).

${ }^{5}$ D. A. Donzis, P. K. Yeung, and K. R. Sreenivasan, Phys. Fluids 20, 045108 (2008).

${ }^{6}$ S. Servidio, W. H. Matthaeus, M. A. Shay, P. A. Cassak, and P. Dmitruk, Phys. Rev. Lett. 102, 115003 (2009).

${ }^{7}$ S. Servidio, W. H. Matthaeus, M. A. Shay, P. Dmitruk, P. A. Cassak, and M. Wan, Phys. Plasmas 17, 032315 (2010).

${ }^{8}$ The kurtosis is the main fourth-order moment discussed here. For a field $j$ with a mean of zero, the kurtosis is defined as $\chi_{j}=\left\langle j^{4}\right\rangle /\left\langle j^{2}\right\rangle^{2}$.

${ }^{9}$ M. Wan, S. Oughton, S. Servidio, and W. H. Matthaeus, Phys. Plasmas 16, 080703 (2009).

${ }^{10}$ T. Stribling and W. H. Matthaeus, Phys. Fluids B 2, 1979 (1990).

${ }^{11}$ U. Frisch, S. Kurien, R. Pandit, W. Pauls, S. S. Ray, A. Wirth, and J.-Z. Zhu, Phys. Rev. Lett. 101, 144501 (2008).

${ }^{12}$ J. W. Bieber, W. Wanner, and W. H. Matthaeus, J. Geophys. Res. 101, 2511, doi:10.1029/95JA02588 (1996).

${ }^{13}$ E. R. Priest and T. G. Forbes, Magnetic Reconnection: MHD Theory and Applications (Cambridge University Press, Cambridge, England, 2000).

${ }^{14}$ A. Pouquet, J. Fluid Mech. 88, 1 (1978).

${ }^{15}$ C. Canuto, M. Y. Hussaini, A. Quarteroni, and T. A. Zang, Spectral Methods in Fluid Mechanics (Springer-Verlag, New York, 1988).

${ }^{16}$ G. S. Patterson and S. A. Orszag, Phys. Fluids 14, 2538 (1971).

${ }^{17}$ T. Y. Hou and R. Li, J. Nonlinear Sci. 16, 639 (2006).

${ }^{18}$ Kurtosis was computed with a time cadence of 0.01 and spectra with a cadence of 0.05 .

${ }^{19}$ U. Frisch, Turbulence (Cambridge University Press, Cambridge, 1995).

${ }^{20}$ This property may not be equivalent to the definitions of intermittency that some researchers require. For example, in connection with higher-order moments and multifractal scaling. Such aspects are not at issue herein, however.

${ }^{21}$ M. Yamada, R. Kulsrud, and H. Ji, Rev. Mod. Phys. 82, 603 (2010).

${ }^{22} \mathrm{P}$. A. Sweet, in Electromagnetic Phenomena in Cosmical Physics, edited by B. Lehnert (Cambridge University Press, New York, 1958), p. 123.

${ }^{23}$ E. N. Parker, J. Geophys. Res. 62, 509, doi:10.1029/JZ062i004p00509 (1957).

${ }^{24}$ Our definition of the $p$-norms is slightly nonstandard since it involves dividing by $C_{X}$.

${ }^{25}$ For example, $\eta=\sqrt{\nu} /\left\langle j^{2}+\omega^{2}\right\rangle^{1 / 4}$ is a reasonable estimate for decaying MHD turbulence with equal resistivity $\mu$ and viscosity $\nu$.

${ }^{26}$ B. R. Pearson, T. A. Yousef, N. E. L. Haugen, A. Brandenburg, and P.-A. Krogstad, Phys. Rev. E 70, 056301 (2004). 